\title{
Aplicação dos modelos para distribuição granulométrica para resíduo de mármore branco
}

\author{
Castro, L. S. ${ }^{1 *}$; Celeri, E. P. ${ }^{2}$; Barañano, A. $\mathrm{G}^{3}$. \\ 1 Programa de Pós - Graduação em Engenharia Química, Universidade Federal de Minas Gerais, Belo Horizonte, MG, \\ Brasil. \\ 2 Graduação em Química , Universidade Federal do Espírito Santo, Alegre, ES, Brasil. \\ 3 Programa de Pós-Graduação em Engenharia Química, Curso de Graduação em Engenharia Química, Departamento \\ de Engenharia Rural, CCA, Universidade Federal do Espírito Santo - UFES, Alegre , ES, Brasil \\ *e-mail: lucienecasttro@hotmail.com
}

\begin{abstract}
Resumo
O resíduo de mármore branco é obtido a partir da extração de rocha ornamental. O presente artigo investigou os modelos matemáticos que descreve a distribuição granulométrica do pó de mármore. O pó de mármore foi seco em uma estufa à $105^{\circ} \mathrm{C}$ durante $2 \mathrm{~h}$, triturados com auxílio de almofariz e pistilo até a obtenção de um pó fino. O peneiramento do pó de mármore foi realizado por conjunto de peneiras de série Tyler com auxílio de agitador magnético de peneiras. A partir das massas foram calculadas as frações mássicas do material e enquanto a granulometria foi obtida a partir da abertura das peneiras. A partir da granulometria foi possível determinar os parâmetros e coeficiente dos modelos matemáticos Gates, Gaudin e Schumann (GGS), Rosin, Rammler e Bennet (RRB) e sigmóide através de ajuste da reta aos dados experimentais. A amostra apresentou diâmetro de partículas de menores que 0,33 $\mathrm{mm}$ até maiores que $1,41 \mathrm{~mm}$, sendo classificado como sólido granulares. Todos os modelos descreveram a distribuição granulométrica do material, no entanto o modelo RRB apresentou melhor ajuste.
\end{abstract}

Palavras Chave: pó de mármore, modelo de distribuição granulométrica.

\begin{abstract}
The residue of white marble is obtained from the extraction of ornamental rock. The present article investigated the mathematical models that describe the granulometric distribution of marble powder. The marble powder residue was dried in an oven at $105^{\circ} \mathrm{C}$ for $2 \mathrm{~h}$, triturated with the aid of mortar and pestle until a fine powder was obtained. The sieving of the marble powder residue was carried out by a set of Tyler series sieves with the aid of a magnetic sieve agitator. From the masses were calculated the mass fractions of the material and while the granulometry was obtained from the opening of the sieves. From the granulometry, it was possible to determine the parameters and coefficient of the mathematical models Gates, Gaudin and Schumann (GGS), Rosin, Rammler and Bennet (RRB) and sigmoid by adjusting the line to the experimental data. The sample had particle diameter of less than $0.33 \mathrm{~mm}$ until greater than $1.41 \mathrm{~mm}$, being classified as granular solid. All the models described the granulometric distribution of the material, but RRB model showed the best adjust.
\end{abstract}

Keywords (Palavras chaves): powder marble, granulometric distribution model .

\section{Introdução}

O mármore é geralmente utilizado nas construções. A indústria de beneficiamento do mármore gera um pó fino, especificamente durante 0 corte e polimento do mármore. Esse resíduo corresponde $25 \%$ da massa total do mármore [1-3].

O Brasil produziu 2 megatoneladas de mármore e travertino, sendo que o estado do Espírito Santo contribuiu com 3.400 .000 toneladas de mármore e travertino [4], portanto quantidade significativa de resíduo são gerados, que pode causar impactos ambientais [1]. Esse resíduo pode ser aplicado na fabricação de telhas, tijolos e ladrilhos [2,3].

O tamanho das partículas e a distribuição dos tamanhos das partículas são fundamentais para várias operações de produção e para o processamento envolvendo materiais particulados. A distribuição do 
tamanho das partículas exerce influência sobre as etapas de produção (transporte, compactação e sinterização) e na microestrutura do material, portanto afeta as propriedades do material (a resistência mecânica, a densidade e as propriedades térmicas e elétricas) [5].

Há diversas técnicas de análise do tamanho das partículas. Dentre essas, destaca-se o peneiramento. $O$ peneiramento ou tamisação é um método de separação de materiais granulados de diferentes tamanhos. Através desse método é possível determinar a granulometria do material. A partir da granulometria do material é possível aplicar os modelos matemáticos tais como foram Gates - Gaudin-Schukman (GGS), Rosin Rammler-Bennet (RRB) e Sigmóide [6-7].

Matté, Silva e Sfredo que avaliaram a aplicação do modelo matemático GGS e RRB para distribuição granulométrica da aveia[8]

Castro e Barañano aplicaram o modelo GGS, RRB e sigmoide para casca de ovo in natura para aplicação como catalisador para biodiesel[9].

Diante disso, o presente trabalho investigou o tamanho de partículas e os modelos matemáticos de distribuição granulométrica para o resíduo de mármore branco para buscar alguns fins para esse resíduo.

\section{Metodologia}

O resíduo de pó de mármore foi seco em uma estufa à $105^{\circ} \mathrm{C}$ durante $2 \mathrm{~h}$, em seguida a amostra foi triturada com auxílio de almofariz e pistilo até a obtenção de um pó fino. A massa de pó de mármore utilizada foi de 100 $\mathrm{g}$, foi adicionada no topo do conjunto de peneiras de série Tyler (16, 20, 32, 35, 48, 60, 80 mesh e fundo). 0 peneiramento foi realizado com auxílio de agitador magnético de peneiras que operou à uma velocidade de frequência $50 \mathrm{rpm}$ e tempo $5 \mathrm{~min}$. $O$ material retido em cada peneira e na panela foi retirado e pesada.

A fração mássica $\left(x_{i}\right)$ foi calculada a partir da massa retida na peneira $(m)$ em relação a massa total $(M)$, o diâmetro das partículas (D) foi obtido a partir da abertura das peneiras, conforme a Equação 1 e 2, respectivamente $[6,7,10]$.

$$
\begin{gathered}
x_{i}=\frac{m}{M} \\
D=\frac{(-) D+(+) D}{2}
\end{gathered}
$$

Nessa equação a simbologia (-) corresponde a massa que atravessou a peneira e já a simbologia (+) corresponde a massa retida na peneira e $X$ é a fração cumulativa.

Os modelos matemáticos para distribuição granulométrica utilizados foram Gates - GaudinSchukman (GGS), Rosin - Rammler-Bennet (RRB) e Sigmóide conforme representados pelas equação 3,4 e 5 [6-7].

$$
\begin{aligned}
& X=\left(\frac{\mathrm{D}}{\mathrm{k}}\right)^{\mathrm{m}} \\
& X=1-\exp \left[-\left(\frac{D}{D^{\prime}}\right)^{n}\right] \\
& X=\frac{1}{1+\left(\frac{\mathrm{k}}{\mathrm{D}}\right)^{\mathrm{m}}}
\end{aligned}
$$

Nessa equação k, $m, n$ e $D^{\prime}$ são os parâmetros a serem ajustados aos dados experimentais.

As equações linearizadas são dadas pela equação 6, 7 e 8 .

$$
\begin{gathered}
\ln \mathrm{X}=\mathrm{m} \ln (D)-\mathrm{m} \ln (k) \\
\ln \left[\ln \left(\frac{1}{1-X}\right)\right]=n \ln (D)-n \ln \left(D^{\prime}\right) \\
\ln \left(\frac{1-X}{X}\right)=m \ln (k)-m \ln (D)
\end{gathered}
$$

Os parâmetros de ajuste aos dados experimentais foram obtido a partir do coeficiente linear e angular da equação da reta do gráfico $\ln \mathrm{X} x \ln (D), \ln \left[\ln \left(\frac{1}{1-X}\right)\right] \times$ $\ln (D)$ e $\ln \left(\frac{1-X}{X}\right) \times \ln (D)$, respectivamente GGS, RRB e sigmóide [6-7].

\section{Discussão dos resultados}

Na tabela 1 está descrito a análise granulométrica do resíduo de mármore branco em pó.

Tabela 1: Distribuição granulométrica

\begin{tabular}{ccccccc}
\hline $\begin{array}{c}\text { Peneira } \\
\text { Tyler } \\
\text { (mesh) }\end{array}$ & $\mathbf{m ~ ( g )}$ & $\left.\mathbf{x}_{\mathbf{i}} \mathbf{\%}\right)$ & $\begin{array}{c}\mathbf{X}_{\mathbf{i}} \\
(\%)\end{array}$ & $\begin{array}{c}(-) \mathbf{D} \\
(\mathbf{m m})\end{array}$ & $\begin{array}{c}(\mathbf{+}) \mathbf{D} \\
(\mathbf{m m})\end{array}$ & $\begin{array}{c}\mathbf{D} \\
(\mathbf{m m})\end{array}$ \\
\hline 16 & 4,74 & 4,77 & 95,23 & - & 0,991 & \\
\hline$-16+20$ & 1,26 & 1,27 & 93,96 & 0,991 & 0,833 & 1,41 \\
\hline$-20+24$ & 1,58 & 1,59 & 92,37 & 0,833 & 0,701 & 1,18 \\
\hline$-24+32$ & 1,62 & 1,63 & 90,74 & 0,701 & 0,495 & 0,95 \\
\hline$-32+35$ & 0,58 & 0,58 & 90,16 & 0,495 & 0,417 & 0,70 \\
\hline$-35+48$ & 2,16 & 2,17 & 87,99 & 0,417 & 0,295 & 0,56 \\
\hline$-48+60$ & 1,67 & 1,68 & 86,31 & 0,295 & 0,248 & 0,42 \\
\hline$-60+80$ & 2,31 & 2,32 & 83,98 & 0,248 & 0,175 & 0,34 \\
\hline Fundo & 83,46 & 83,98 & 0,00 & fundo & Fundo & fundo \\
\hline Total & 99,377 & 100,00 & & & & \\
\hline
\end{tabular}

Observou que a maior parte da amostra $(83,46 \%)$ ficou no fundo da peneira que evidencia que a essa porção da amostra apresentou diâmetro de partículas menores que $0,34 \mathrm{~mm}$. O diâmetro de partículas da amostra variou de menores que $0,33 \mathrm{~mm}$ até maiores que 1,41 $\mathrm{mm}$, sendo classificado como sólido granulares. A minoria da amostra (0,58\%) apresentou diâmetro de partículas de $0,70 \mathrm{~mm}$.

A figura 2 mostra os modelos matemáticos para distribuição granulométrica. 

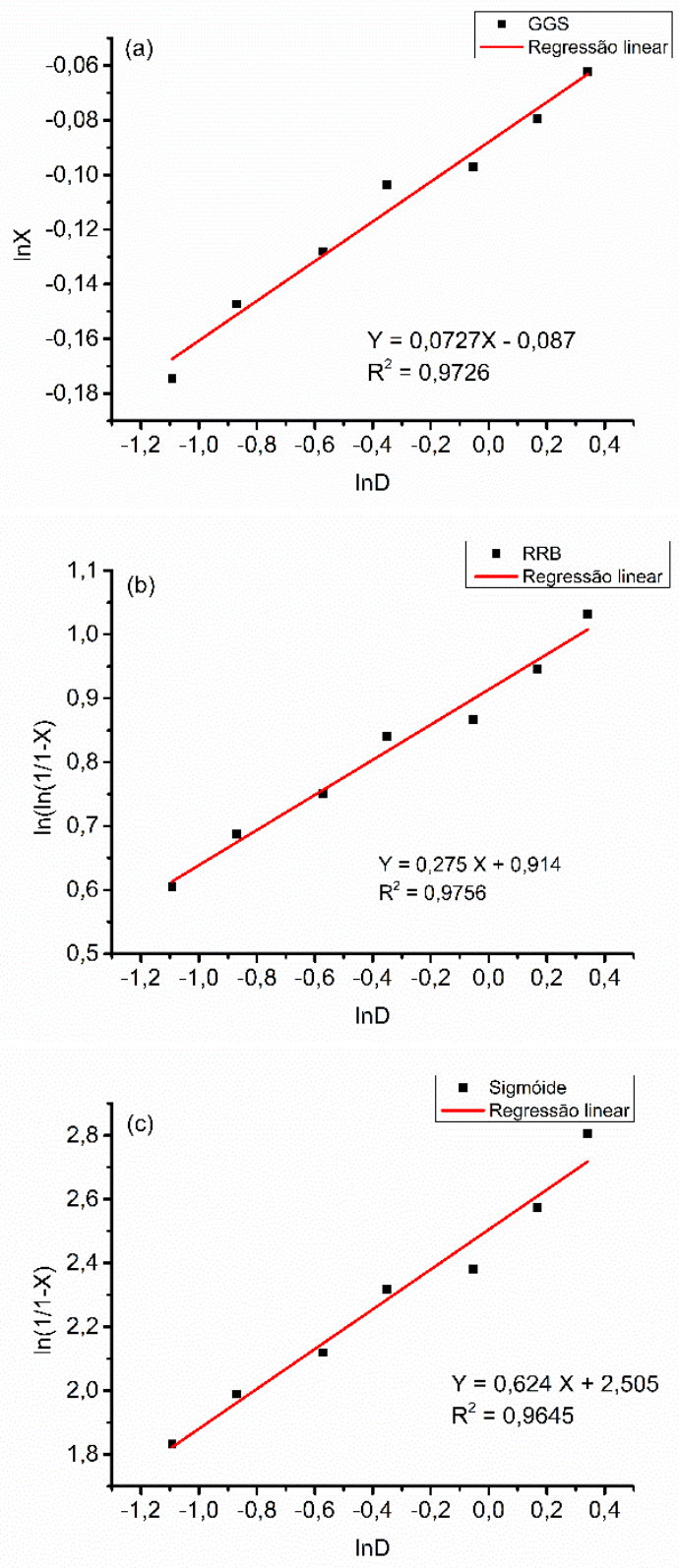

Figura 2 - Modelos matemáticos de distribuição granulométrica (a) GGS (b) RRB e (c) Sigmóide.

A tabela 2 mostra as equações características dos modelos matemáticos e o coeficiente de correlação $\left(R^{2}\right)$ foram obtidos a partir da figura 2. Os três modelos ajustaram satisfatoriamente os dados, o modelo RRB apresentou maior coeficiente de correlação, portanto, melhor ajuste. Ao contrário de Matté, Silva e Sfredo que avaliaram a aplicação do modelo matemático GGS e RRB para distribuição granulométrica da aveia e concluíram que o melhor modelo foi GGS. Esse resultado foi semelhante ao obtido por Castro e Barañano [9] que avaliaram a distribuição granulométrica de casca ovo e observaram que 0 melhor modelo é RRB.

\begin{tabular}{c|c|c}
\multicolumn{3}{c}{ Tabela 2: Equações dos modelos matemáticos. } \\
\hline $\begin{array}{c}\text { Modelo } \\
\text { matemático }\end{array}$ & Equação & $\mathbf{R}^{2}$ \\
\hline GGS & $\mathrm{X}=\left(\frac{\mathrm{D}}{0,2980}\right)^{0,072}$ & 0,9726 \\
\hline RRB & $X \quad 1-\exp \left[-\left(\frac{D}{0,036}\right)^{0,2751}\right]$ & 0,9756 \\
\hline Sigmóide & $\mathrm{X}=\frac{1}{1+\left(\frac{0,1539}{\mathrm{D}}\right)^{0,6248}}$ & 0,9645 \\
\hline
\end{tabular}

$O$ valor de $m$ de 0,072 , portanto, $m<1$, logo evidencia que o material não apresenta o tamanho das partículas uniforme. O parâmetro $k$ corresponde ao diâmetro médio das partículas tal que $100 \%$ da massa da amostra corresponde a partículas que $\mathrm{k}$, ou seja, menor que $0,298 \mathrm{~mm}$.

O valor de $n$ de 0,275 , logo $n<1$. O parâmetro ' D' corresponde ao diâmetro médio das partículas tal que $63,2 \%$ da massa da amostra corresponde a partículas que $\mathrm{k}$, ou seja, menor que $0,036 \mathrm{~mm}$.

O parâmetro k para o modelo sigmoide corresponde ao diâmetro médio das partículas tal que $50 \%$ da massa da amostra corresponde a partículas que $\mathrm{k}$, ou seja, menor que $0,154 \mathrm{~mm}$ e o valor de $\mathrm{m}>1$.

\section{Conclusão}

O resíduo de mármore foi classificado em peneiras de abertura de 16 a 80 mesh, sendo que a maior parte da amostra apresentou um diâmetro das partículas inferiores a 0,33 $\mathrm{mm}$.

Todos os modelos descreveram a distribuição granulométrica, no entanto o modelo RRB apresentou melhor ajustes para o material em estudo.

\section{Agradecimentos}

Os autores agradecem a CNPq pelo apoio financeiro.

\section{Referências}

[1] DEMIREL, B. Int. J. Phy. Sci., v 5, p 1372-1380, 2010.

[2] ALMEIDA, T. F. Reaproveitamento de resíduo de pó de mármore e chamote na produção de material cerâmico para isolação térmica.. 102f. Dissertação (Mestrado em Engenharia e Ciências dos Materiais), Programa de Pós-Graduação em Engenharia e Ciências dos Materiais, Universidade Estadual do Norte Fluminense, Campos dos Goycatazes, 2014.

[3] FREITAS, J. J. G., RAYMUNDO, V., JESUS, H. C. Revista Brasileira de Geociências, 42(3), 2012. 
[4] ABIROCHAS. Produção brasileira de lavra. Disponíveis em: < http://abirochas.com.br/wpcontent/uploads/2018/06/Panorama/Producao_Brasileir a_Lavra_b.pdf >. Acesso: 31 jul 2019.

[5] PAPINI, C. J. Estudo comparativo de métodos de determinação do tamanho de partícula. Dissertação (Mestrado em Ciências), Programa de Pós-Graduação em Ciências, Universidade São Paulo, São Paulo, 2003.

[6] CREMASCO, M. A. Operações unitárias em sistemas particulados e fluidomecânicos. São Paulo: Blucher, 2012.

[7] MASSARANI, G. Fluidodinâmica em Sistemas Particulados. Rio de Janeiro: e-papers, 2002.

[8] MATTÉ, L. S., SILVA, G S.; SFREDO, M. A. Estudo da aplicação de modelos matemáticos para a distribuição granulométrica de aveia em flocos finos. In: Encontro de Educação, Ciência e Tecnologia do IFRS
Câmpus Erechim, 2, 2017, Erechim. Anais eletrônicos...Disponível em: <http://erechim.ifrs.edu.br/site/midias/arquivos/2014101 713118708poster_iii_jepex_estudo_da_aplicacao_de _modelos_matematicos.pdf $>$. Acesso 09 de Agosto de 2017.

[9] CASTRO, L. S; BARAÑANO, A. G. Aplicação dos modelos matemáticos na distribuição granulométrica da casca de ovo. In: Encontro de Física Aplicada, 8, 2017, Anais eletrônicos...Disponível em: https://www.proceedings.blucher.com.br/articledetails/aplicao-dos-modelos-matemticos-na-distribuiogranulomtrica-da-casca-de-ovo-27749. Acesso : 20 de Abril de 2019.

[10] FOUST, A. et al. Princípios de operações. Rio de Janeiro: LTC, 1982.

[11] 